\title{
Unparticles in diphoton production to NLO in QCD at the LHC
}

\author{
M. C. $\operatorname{Kumar}^{a, b}{ }_{1}, \operatorname{Prakash}_{\operatorname{Mathews}^{a}}{ }_{2}, \mathrm{~V}, \operatorname{Ravindran}^{c}$ 3, Anurag $\operatorname{Tripathi}^{c} 4_{4}$ \\ a) Saha Institute of Nuclear Physics, 1/AF Bidhan Nagar, Kolkata 700 064, India. \\ b) School of Physics, University of Hyderabad, Hyderabad 500 046, India. \\ c)Regional Centre for Accelerator-based Particle Physics, Harish-Chandra Research \\ Institute, Chhatnag Road, Jhunsi, Allahabad, India.
}

\begin{abstract}
We compute to next-to-leading order in QCD the tensor unparticle contribution to the diphoton production at the LHC, wherein the unparticle sector is a consequence of (a) scale invariance but not full conformal invariance and (b) conformal invariance. We use the semi-analytical two cutoff phase space slicing method to handle the $\mathcal{O}\left(\alpha_{s}\right)$ corrections to the $p p \rightarrow \gamma \gamma X$ and show that our results are insensitive to the soft and collinear cutoffs. In order to avoid the contribution of the photons due to fragmentation, we employ the smooth cone isolation criterion. Significance of the QCD corrections to the diphoton events including unparticles is highlighted.
\end{abstract}

PACS numbers: 12.60.-i, 11.25.Hf, 14.80.-j

\footnotetext{
${ }^{1}$ mc.kumar@saha.ac.in

2 prakash.mathews@saha.ac.in

3 ravindra@mri.ernet.in

4anurag@mri.ernet.in
} 


\section{Introduction}

Diphoton signals play very important role in the phenomenological studies of the standard model (SM) and also of beyond the standard model (BSM). These photons can constitute a potential background to those coming from Higgs decay. In the BSM, namely, supersymmetric [1], extra-dimensional [2] and unparticle (UP) models [3] the diphoton productions can constrain the model parameters. Hence it is important to have precise predictions [4-6] for this process in the theory in order to discover the Higgs boson as well as to unravel physics beyond the standard model. Recently, unparticle

physics $[7,8]$ has gained a lot of attention in the context of collider signatures aiming to study new physics signals. In a recent paper [3], it was found that the diphoton production at the hadron colliders is an important process to look for effects coming from unparticle physics. This study was based on a leading order (LO) computation in QCD. Since the gluons contribute even at LO in the unparticle scenario, the higher order QCD effects are expected to play a significant role in the predictions. In this paper we report contributions to next-to-leading order (NLO) in QCD, coming from a model with tensor unparticles as well as from the SM.

Parton level cross sections beyond the LO in perturbation theory encounter IR singularities resulting from the soft gluons and massless collinear partons. In addition to the above singularities resulting from partons, the photons in the final states can also give collinear singularities when they become collinear to one of the final state partons. These collinear singularities, often called QED singularities, can be avoided by appropriately constructing observables that are insensitive to them. In addition, a parton in the final state can fragment non-perturbatively into a photon and a bunch of hadrons collinear to it, parameterized by photon fragmentation functions [9]. The QED singularities can be removed by either absorbing them into these fragmentation functions or by suitable definition of diphoton events that removes these contributions. The approach involving fragmentation functions is theoretically tedious and also brings in additional non-perturbative input that is poorly known to date. Hence we adopt an 
alternate smooth cone isolation criterion proposed by Frixione [10] which ensures that the fragmentation contribution and the final state QED singularities are suppressed.

\section{Unparticle physics}

In a recent paper, Georgi $[7,8]$ considered a scenario wherein the SM is weakly coupled to a sector which is scale invariant below an IR scale $\Lambda_{\mathcal{U}}$. Consequently conventional particle interpretation at low energies in this new sector fails. These fields were termed as unparticles by Georgi. The unparticles could be interpreted as fractional number of massless particles [7] or a collection of particles with a particular mass distribution [11] as a result of deconstruction. The coupling of these unparticles to the SM would lead to direct phenomenological consequences due to the peculiar unparticle phase space [7] and propagator $[8,13]$, which are determined by scale invariance. Their coupling to the SM Higgs could lead to a constraint on the unparticle sector due to breaking of the scale invariance when the Higgs gets a vacuum expectation value [12]. As a consequence unparticle physics may be relevant at the high energy colliders which have been actively investigated $[3,7,8,13-15]$. In [16], the corrected propagator and lower bounds on the scaling dimensions of the vector and the tensor operators were obtained using unitarity constraints on scattering amplitudes. In this paper, we use these new corrected results to do our phenomenology.

The unparticle operators could be of scalar, vector, tensor or fermionic type. In the present article we restrict ourselves to tensor unparticles [3,14] coupled to SM fields given by

$$
\frac{\lambda_{t}}{\Lambda_{\mathcal{U}}^{d_{\mathcal{U}}}} T_{\mu \nu} O_{\mathcal{U}}^{\mu \nu}
$$

where $\lambda_{t}$ is the dimensionless coupling constant for the unparticle tensor operator $O_{\mathcal{U}}^{\mu \nu}$ which is traceless and symmetric and has a scaling dimension $d_{\mathcal{U}} . T_{\mu \nu}$ is the energy momentum tensor of the SM. Scale invariance restricts the scaling dimension of tensor unparticle operator to $d_{\mathcal{U}} \geq 3$ [16]. Conformal invariance on the other hand leads to a constraint $d_{\mathcal{U}} \geq 4$ on the second rank tensor operators. Scale and conformal symmetries 
can only guide us on fixing the tensor structures of the propagator leaving the overall normalisation undetermined. Unlike the conformal invariance, the scale invariance does not fix the relative coefficients of the tensors appearing in the tensor propagator [16]. We use the following propagator for our phenomenological study:

$$
\begin{aligned}
\int d^{4} x e^{i k \cdot x}\left\langle 0\left|T O_{\mathcal{U}}^{\mu \nu}(x) O_{\mathcal{U}}^{\alpha \beta}(0)\right| 0\right\rangle= & -i C_{T} \frac{\Gamma\left(2-d_{\mathcal{U}}\right)}{4^{d_{\mathcal{U}}-1} \Gamma\left(d_{\mathcal{U}}+2\right)}\left(-k^{2}\right)^{d_{\mathcal{U}}-2} \\
& \times\left[d_{\mathcal{U}}\left(d_{\mathcal{U}}-1\right)\left(g_{\mu \alpha} g_{\nu \beta}+\mu \leftrightarrow \nu\right)+\ldots\right]
\end{aligned}
$$

where we have chosen $C_{T}=1$. The terms given by ellipses do not contribute to the diphoton production. The terms in the ellipses depend on tensors, proportional to $g^{\mu \nu}, k^{\mu}$ and $k^{\nu}$. The exact tensorial form of course depends on the symmetry (scale or conformal). These terms do not contribute to physical processes thanks to the conservation and traceless nature of the SM energy momentum tensor. Hence the symmetry restriction enters only through the scaling dimension $d_{\mathcal{U}}$ (the overall undetermined constant could be different for the scale and conformal invariant propagators). Hence we can safely use the above propagator Eq. (21) with $d_{\mathcal{U}} \geq 3(\geq 4)$ for scale (conformal) invariant analysis. As larger $d_{\mathcal{U}}$ values give smaller unparticle contributions, we would demand only scale invariance which allows smaller values of $d_{\mathcal{U}}$. In addition, as the SM energy momentum tensor is a conserved quantity, it does not require any operator mixing under SM renormalisation.

For our study, we closely followed Georgi's approach [7] to fix the coefficient of the effective interaction term at the weak scale for our phenomenology. Accordingly, the effective interaction at the weak scale is proportional to

$$
C_{\mathcal{U}} \frac{\Lambda_{\mathcal{U}}^{d_{B Z}-d_{\mathcal{U}}}}{M_{\mathcal{U}}^{k}},
$$

where $M_{\mathcal{U}}$ is the mass of heavy mediators in the hidden sector, $\Lambda_{\mathcal{U}}$ is the scale at which dimensional transmutation occurs and $C_{\mathcal{U}}$ is the Wilson coefficient. $d_{B Z}$ and $d_{\mathcal{U}}$ are the scaling dimensions of Banks-Zaks (BZ) [17] and the unparticle operators respectively. In the case of tensor unparticle operator coupled to the energy momentum tensor of the SM fields (having scaling dimension 4), the combination $C_{\mathcal{U}} \Lambda_{\mathcal{U}}^{d_{B Z}} / M_{\mathcal{U}}^{k}$ becomes dimensionless, 
which we call $\lambda_{t}$ in Eq. 1. This can be phenomenologically interesting only if we assume the dimensional transmutation scale closer to the electroweak scale and hence we choose $\Lambda_{\mathcal{U}}$ to be of the order of $\mathrm{TeV}$.

\section{Diphotons at hadron colliders}

The hadronic cross section, $d \sigma^{P_{1}, P_{2}}$, in the QCD improved parton model can be expressed in terms of mass factorized parton level subprocess cross sections, $\Delta_{a b}$ convoluted with PDFs $f_{a / P}$ and $f_{b / P}$ :

$$
d \sigma^{P_{1}, P_{2}}(S)=\sum_{a, b=q, \bar{q}, g} \int d x_{a} \int d x_{b} f_{a / P_{1}}\left(x_{a}, \mu_{F}^{2}\right) f_{b / P_{2}}\left(x_{b}, \mu_{F}^{2}\right) \Delta_{a b}\left(x_{a}, x_{b}, S, \mu_{F}^{2}\right),
$$

where $x_{a}, x_{b}$ are the momentum fractions of the incoming partons $a$ and $b$ respectively, $S$ is the invariant mass square of the hadronic system and $\mu_{F}$ is the factorization scale. Various kinematic constraints on the final states can be applied by introducing delta functions of these constraints. At LO in QCD we have contributions of order $\alpha^{2}, \alpha \lambda_{t}^{2}, \lambda_{t}^{4}$. Here $\alpha$ is the fine structure constant. At this order, the diphoton signal in the SM comes from the process $q+\bar{q} \rightarrow \gamma+\gamma$ and in the unparticle physics we have $q+\bar{q} \rightarrow \gamma+\gamma$ and $g+g \rightarrow \gamma+\gamma$. The presence of the gluon initiated process at LO in QCD is due to the fact that the unparticle fields couple to quarks and gluons with the same strength. At NLO, contributions are of the order $\alpha_{s} \alpha^{2}, \alpha_{s} \alpha \lambda_{t}^{2}$, and $\alpha_{s} \lambda_{t}^{4}$. In the SM, $q+\bar{q} \rightarrow \gamma+\gamma+$ one loop, $q+\bar{q} \rightarrow \gamma+\gamma+g$ and $q(\bar{q})+g \rightarrow \gamma+\gamma+q(\bar{q})$ contribute. In the unparticle case, in addition to the above processes, $g+g \rightarrow \gamma+\gamma+$ one loop and $g+g \rightarrow \gamma+\gamma+g$, also contribute. In addition, at the NLO SM $g g \rightarrow \gamma \gamma$ box diagram contributes via its interference with leading order $g g \rightarrow \gamma \gamma$ diagram with an unparticle propagator.

Before we compute the observable with the desired final state kinematical constraints, we compute the reduced cross sections $\left(\Delta_{a b}\right.$ in Eq. (44) $)$ that are free of both IR and ultraviolet singularities. The UV divergences resulting from the processes involving loops are regulated using dimensional regularization and renormalized in the $\overline{M S}$ scheme. 
To study various kinematical distributions of these diphoton events with the experimental cuts, a fully analytical computation at the NLO level is tedious. Hence we have opted for a semi-analytical approach, namely, a two cutoff phase-space slicing method [18]. Here, the phase space integrals are split into regions that are sensitive to soft and collinear singularities and those that are free of them. Such a slicing of the phase space is done using two very small parameters denoted by $\delta_{s}$ and $\delta_{c}$ which define the boundaries of soft and collinear regions respectively. Processes involving real gluons or quark/antiquark emissions can be decomposed as

$$
d \sigma_{a b}^{r e a l}=d \sigma_{a b, s o f t}^{r e a l}\left(\delta_{s}\right)+d \sigma_{a b, c o l}^{r e a l}\left(\delta_{s}, \delta_{c}\right)+d \sigma_{a b, f i n}^{r e a l}\left(\delta_{s}, \delta_{c}\right)
$$

The subscripts col and fin correspond to collinear and finite parts respectively. In the above equation, $d \sigma_{a b, s o f t}^{\text {real }}\left(\delta_{s}\right)$ is the cross section wherein the phase space integrals of the out going gluons are constrained such that the energy of the gluon in the CM frame of the incoming partons, is in the range $0 \leq E_{g} \leq \delta_{s} \sqrt{s_{a b}} / 2 . s_{a b}$ is the invariant mass squared of the incoming partons $a$ and $b$. Similarly, $d \sigma_{a b, c o l}^{r e a l}\left(\delta_{s}, \delta_{c}\right)$ is the cross section where the collinear regions of the final state partons are integrated out keeping the invariant mass squared of the pair of collinear partons to be less than $\delta_{c} s_{a b}$. The remaining cross section where the soft and collinear regions are removed is denoted by $d \sigma_{a b, f i n}^{\text {real }}\left(\delta_{s}, \delta_{c}\right)$ and can be evaluated in 4-dimensions itself. The integrals involving soft and collinear regions of the phase space are regulated using dimensional regularization and can be computed analytically. The next step involves the computation of the virtual gluon corrections denoted by $\sigma_{a b}^{V}$ to the born processes to NLO in QCD. The soft and collinear singularities present in the loop integrals of these virtual corrections are regulated using dimensional regularization. The soft and collinear singularities coming from the phase space as well as from the loop integrals manifest themselves as poles in $\varepsilon$. One can easily show that the following combination

$$
d \sigma_{a b}^{S+V}\left(\delta_{s}\right) \equiv d \sigma_{a b, s o f t}^{r e a l}\left(\delta_{s}\right)+\sigma_{a b}^{V}
$$

is free of $\varepsilon$ poles coming from the soft gluons. The collinear singularities from the $\sigma_{a b}^{V}$ 
and $d \sigma_{a b, c o l}^{r e a l}\left(\delta_{s}, \delta_{c}\right)$ will go away if the mass factorization counter terms denoted by $d \sigma_{a b}^{F}$, defined in the $\overline{M S}$ scheme, are included in the cross sections. The combination

$$
d \sigma_{a b}^{S+V+C+F}\left(\delta_{s}, \delta_{c}\right)=d \sigma_{a b}^{S+V}\left(\delta_{s}\right)+d \sigma_{a b, c o l}^{r e a l}\left(\delta_{s}, \delta_{c}\right)+d \sigma_{a b}^{F}
$$

is free of all IR singularities in QCD. Finally we end up with IR finite cross section given by

$$
\Delta_{a b}=d \sigma_{a b}^{S+V+C+F}\left(\delta_{s}, \delta_{c}\right)+d \sigma_{a b, f i n}^{r e a l}\left(\delta_{s}, \delta_{c}\right)
$$

Even though $d \sigma_{a b}^{S+V+C+F}$ and $d \sigma_{a b, f i n}^{r e a l}$ are separately dependent on $\delta_{s}$ and $\delta_{c}$, their sum is expected to be independent of these parameters. Compact and simple analytical expressions for soft and collinear parts of the cross sections are reserved for a detailed publication [19].

The reduced cross section Eq. (8) is IR finite. The additional final state QED singularities coming from the SM subprocess $q(\bar{q}) g \rightarrow \gamma \gamma q(\bar{q})$ are suppressed by the smooth cone isolation criteria advocated by Frixione [10]. The isolation criteria on each of the photons is imposed by using the longitudinal boost invariant dimensionless parameter $R_{i \gamma}=\sqrt{\left(\eta_{i}-\eta_{\gamma}\right)^{2}+\left(\phi_{i}-\phi_{\gamma}\right)^{2}}$ where $\eta_{i}$ and $\eta_{\gamma}$ are pseudo-rapidities of the outgoing hadron $i$ and the photon respectively. Similarly, $\phi_{i}$ and $\phi_{\gamma}$ are their azimuthal angles with respect to the beam direction. Within a circle of radius $R_{0}$ centered at each photon we reject any event unless the following condition is fulfilled:

$$
\sum_{i} E_{T, i} \theta\left(R-R_{i \gamma}\right) \leq \mathcal{H}(R), \quad \text { for } \text { all } R \leq R_{0}
$$

where $E_{T, i}$ is the transverse energy of the hadron $i$. The function $\mathcal{H}(R)$ must vanish as $R$ goes to zero in order to get IR finite observable. One such choice put forth by Frixione is

$$
\mathcal{H}(R)=E_{T}^{i s o}\left(\frac{1-\cos (R)}{1-\cos \left(R_{0}\right)}\right)^{n}
$$

where $E_{T}^{i s o}$ is a fixed energy and $n \geq 1$. 
Since the isolation criteria on diphoton events does not allow any hard partonic (hadronic) activity closer (collinear) to either of the photons, the fragmentation contributions which are collinear in nature are suppressed. The isolation criteria also ensures the cancellation of the soft singularities coming from the real and virtual gluons as it does not restrict the phase space of the soft gluons. By imposing finite transverse momentum cuts on the isolated photons, the smooth cone isolation criteria does not reject any hard parton which could be collinear to the initial states - ensuring mass factorization. Hence the isolation criteria on the diphotons guarantees the use of the phase space slicing method to compute an IR safe observable. In addition, we can safely employ the experimental cuts on the rapidity $\left|y^{\gamma}\right|$ of the photons.

\section{Results}

In this section we present our results for $\sqrt{S}=14 \mathrm{TeV}$ at the LHC. The virtual and real corrections have been evaluated in the limit of vanishing quark masses. We have evaluated LO and NLO cross sections with CTEQ6L and CTEQ6M parton density sets [20] with the corresponding strong coupling constant $\alpha_{s}\left(M_{Z}\right)=0.118$. The fine structure constant is $\alpha=1 / 128$. A single scale $Q$, the invariant mass of the photon pair, is used for the renormalization and factorization scale. The scale $\Lambda_{\mathcal{U}}$ at which scale invariance sets in the $\mathrm{BZ}$ sector is chosen to be $1 \mathrm{TeV}$. Scale invariance restricts the scaling dimension of tensor operators to $d_{\mathcal{U}} \geq 3$, in our calculation we have chosen $d_{\mathcal{U}}=3.01$. The coupling $\lambda_{t}$ is taken to be of order one.

We have imposed the kinematical cuts on the photons as used by the ATLAS detector [21]: $p_{T}^{\gamma}>40(25) \mathrm{GeV}$ for harder (softer) photon, $\left|\eta_{\gamma}\right|<2.5$ for each photon. The photons are restricted to have a separation of at least $R_{\gamma \gamma}=0.4$. In addition to these constraints, we impose smooth cone isolation criterion on photons given in Eq. (8). $R_{0}=0.4$ is taken for the cone radius. Unless otherwise specified we use $n=2$ and $E_{T}^{i s o}=15 \mathrm{GeV}$ which appear in $\mathcal{H}(R)$

To show that the results are independent of the choice of the slicing parameters 

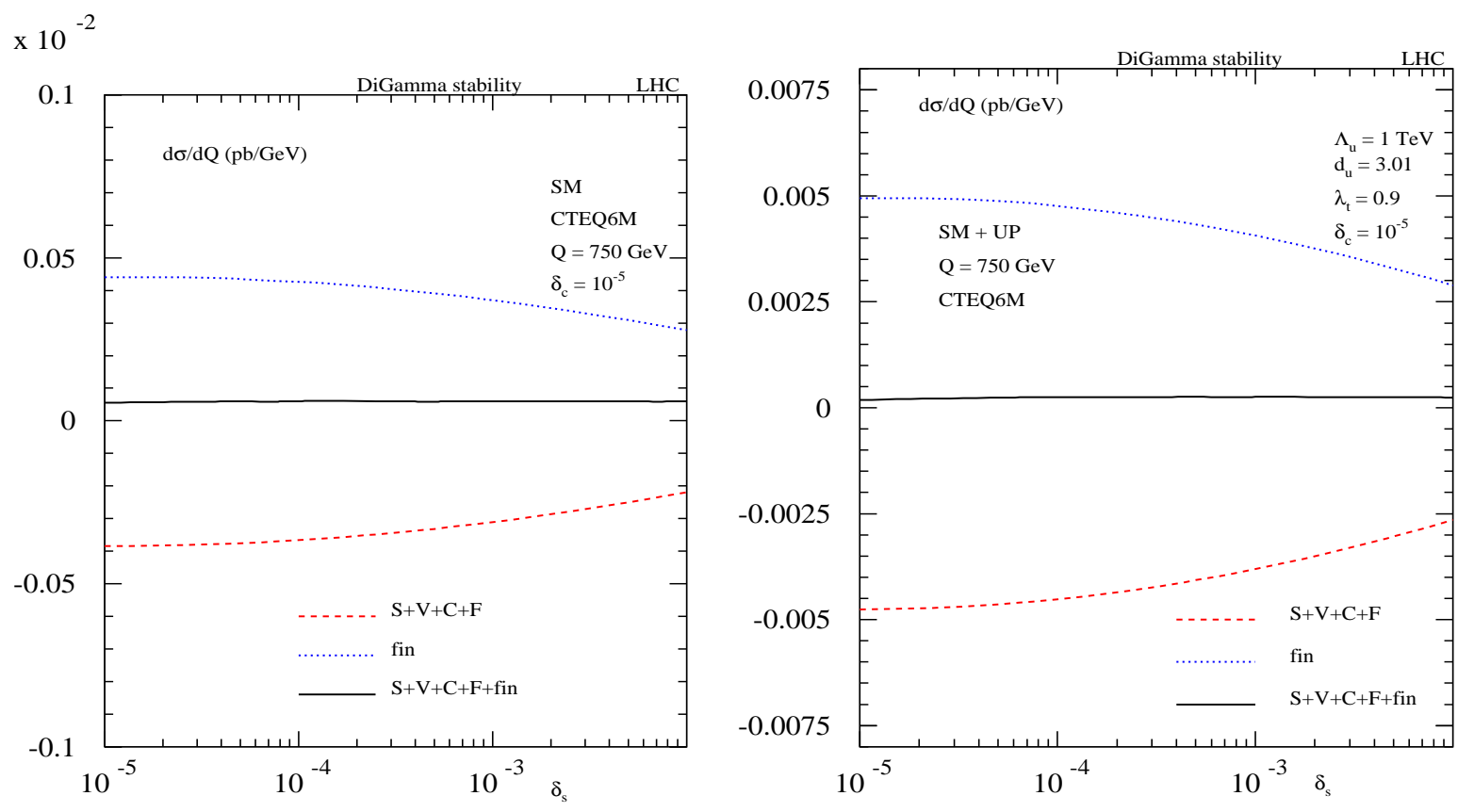

Figure 1: Plots showing stability of $d \sigma / d Q$ for the SM (left panel) and the SM+ UP (right panel) against $\delta_{s}$ variation with the choice of $\delta_{c}=10^{-5}$.

$\delta_{s}$ and $\delta_{c}$, we have plotted $d \sigma_{a b}^{S+V+C+F}\left(\delta_{s}, \delta_{c}\right)$ and $d \sigma_{a b, f i n}^{r e a l}\left(\delta_{s}, \delta_{c}\right)$ pieces of $d \sigma / d Q$ as functions of $\delta_{s}$ with $\delta_{c}$ kept fixed at a very small value $10^{-5}$ in Fig. 1. We see that the sum is fairly stable under the variation. The variation is around $2 \%$ for $\mathrm{SM}$ and is less then $5 \%$ for the signal $(\mathrm{SM}+\mathrm{UP})$. For rest of our numerical study, we have chosen $\delta_{s}=10^{-3}$ and $\delta_{c}=10^{-5}$. As SM NLO results exist in literature [4-6], to further check our code we have compared our results with [5] using their isolation criterion $\left[\mathcal{H}(R)=p_{T}(\gamma) \epsilon\left([1-\cos (R)] /\left[1-\cos \left(R_{0}\right)\right]\right)^{n}\right]$ and their choice of $\mu_{F}, \mu_{R}$ and PDFs. We have found good agreement with [5].

We present various subprocess contributions to NLO in QCD in the invariant mass distribution for the range $400<Q<900 \mathrm{GeV}$ in Fig. 2. In the SM both $q \bar{q}$ and $q g$ subprocess contributions are positive with $q \bar{q}$ contribution being dominant over that of $q g$ for the range of $Q$ considered. However for smaller values of $\mathrm{Q}(<150 \mathrm{GeV}), q g$ contribution will be dominant due to the large gluon flux. In the unparticle sector the $q \bar{q}$ and $g g$ subprocess contributions via the pure unparticle exchange (direct) are positive 


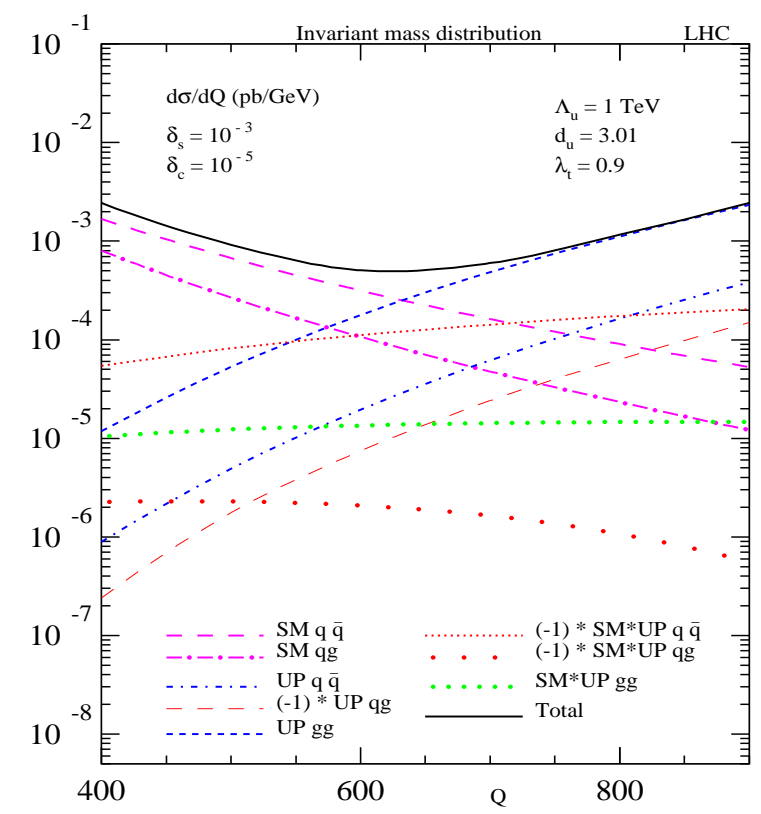

Figure 2: Subprocess contributions in the SM and unparticle model in the invariant mass distribution at NLO for $400<Q<900 \mathrm{GeV}$.

while the $q g$ contribution is negative. At the interference level the $g g$ interference with the SM box has a positive contribution and is almost constant for the range of $Q$ considered. However, the interference of both $q \bar{q}$ and $q g$ subprocesses with the SM have negative contributions and are larger in magnitude compared to the direct ones. As a result, the total $\mathcal{U}$-particle contributions (direct+interference) can have a pattern that will change the sign at some high value of $Q$. Consequently, below this $Q$ the signal can be lower than the SM background. The direct $\mathcal{U}$-particle exchange contributions can become significant in the large $Q$ region because the cross sections go as powers of $Q / \Lambda_{\mathcal{U}}$, thus leading to the visibility of the $\mathcal{U}$-particles only in that region of $Q$. It is worth noting that only the $g g$ initiated subprocess has the dominant contribution over the rest in this region.

In Fig. 3, we present our results for $d \sigma / d Q$ (left panel) for $Q$ between 400 and $900 \mathrm{GeV}$ and $d \sigma / d Y$ (right panel) for $|Y|<2.0$, where $Y$ is the rapidity of the diphoton system. The short dashed (LO) and the long dashed lines (NLO) correspond to SM distributions. 

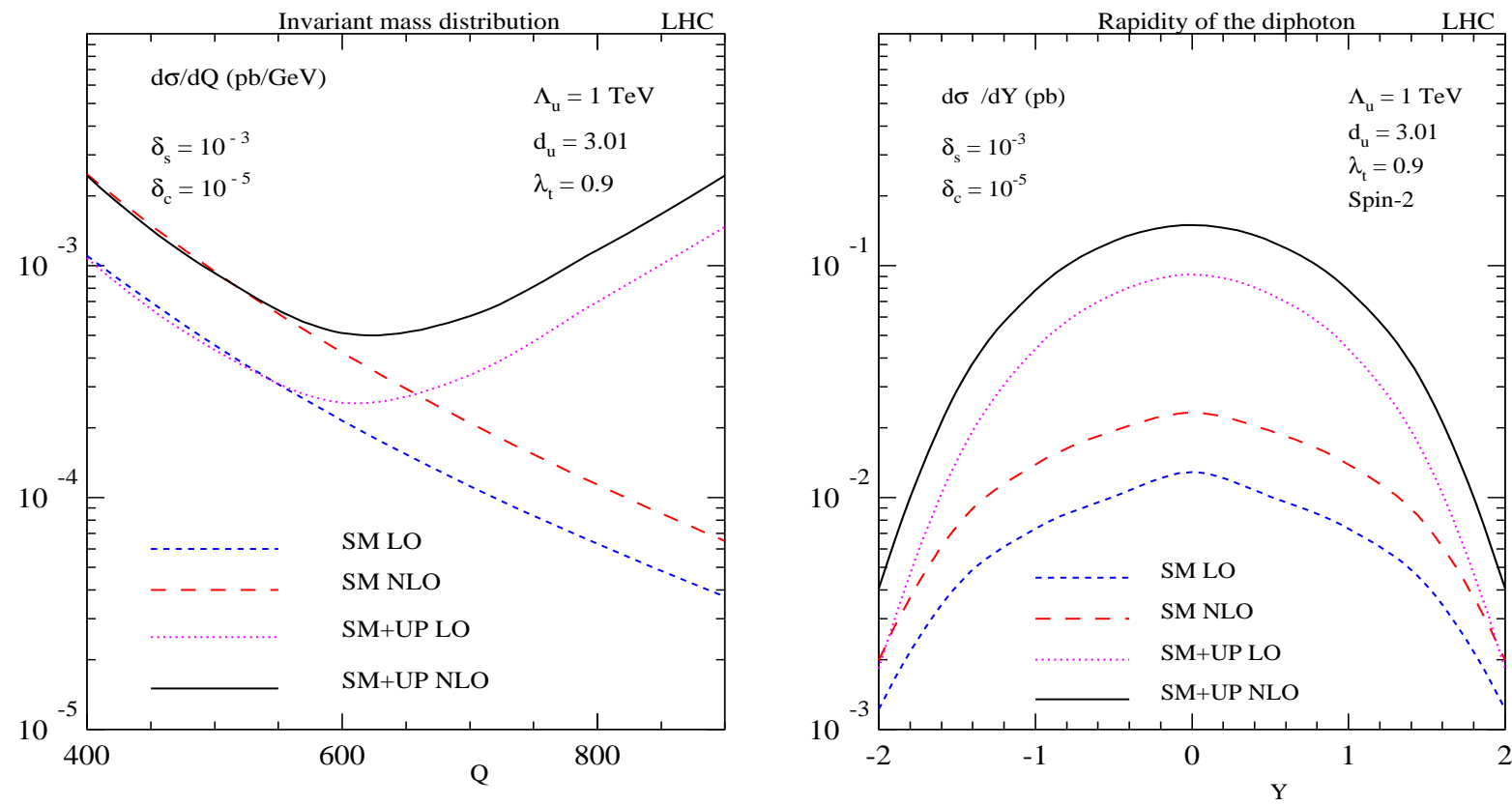

Figure 3: Plots showing invariant mass (left panel) and rapidity (right panel) distributions of the diphoton system with $d_{\mathcal{U}}=3.01, \Lambda_{\mathcal{U}}=1 \mathrm{TeV}$ and $\lambda_{t}=0.9$. For rapidity distribution $Q$ is integrated in the range $600 \mathrm{GeV}<Q<0.9 \Lambda_{\mathcal{U}}$.

The dotted (LO) and the solid (NLO) lines correspond to the signal (SM+UP) of the unparticle physics. As expected we find that the unparticle contribution to the invariant mass distribution grows with $Q$ and it dominates over the $\mathrm{SM}$ contributions above $Q=$ $600 \mathrm{GeV}$. The precise value where this happens will depend very much on the choice of $\Lambda_{\mathcal{U}}$ and other unparticle parameters. Since unparticle effects can be seen in the larger values of $Q$, rapidity distributions are computed by integrating $Q$ between 600 and 900 $\mathrm{GeV}$. Near the central value of $Y$, we find large enhancement of the cross section from the SM results if we include unparticle contributions.

At next-to-leading order the total transverse energy of the hadrons is due to a single parton around the photons and does not correspond to the actual hadronic energy in an experiment. Hence the $E_{T}^{i s o}$ at the parton level is a crude estimate of that of the jets at the detector level. To show the dependence on $E_{T}^{i s o}$ we present the invariant mass distribution for $E_{T}^{i s o}=15 \mathrm{GeV}$ and $E_{T}^{i s o}=30 \mathrm{GeV}$, for $n=2$. To study the dependence of our predictions on the choice of $\mathcal{H}(R)$ we have varied it by changing $n$ from 1 to 2 

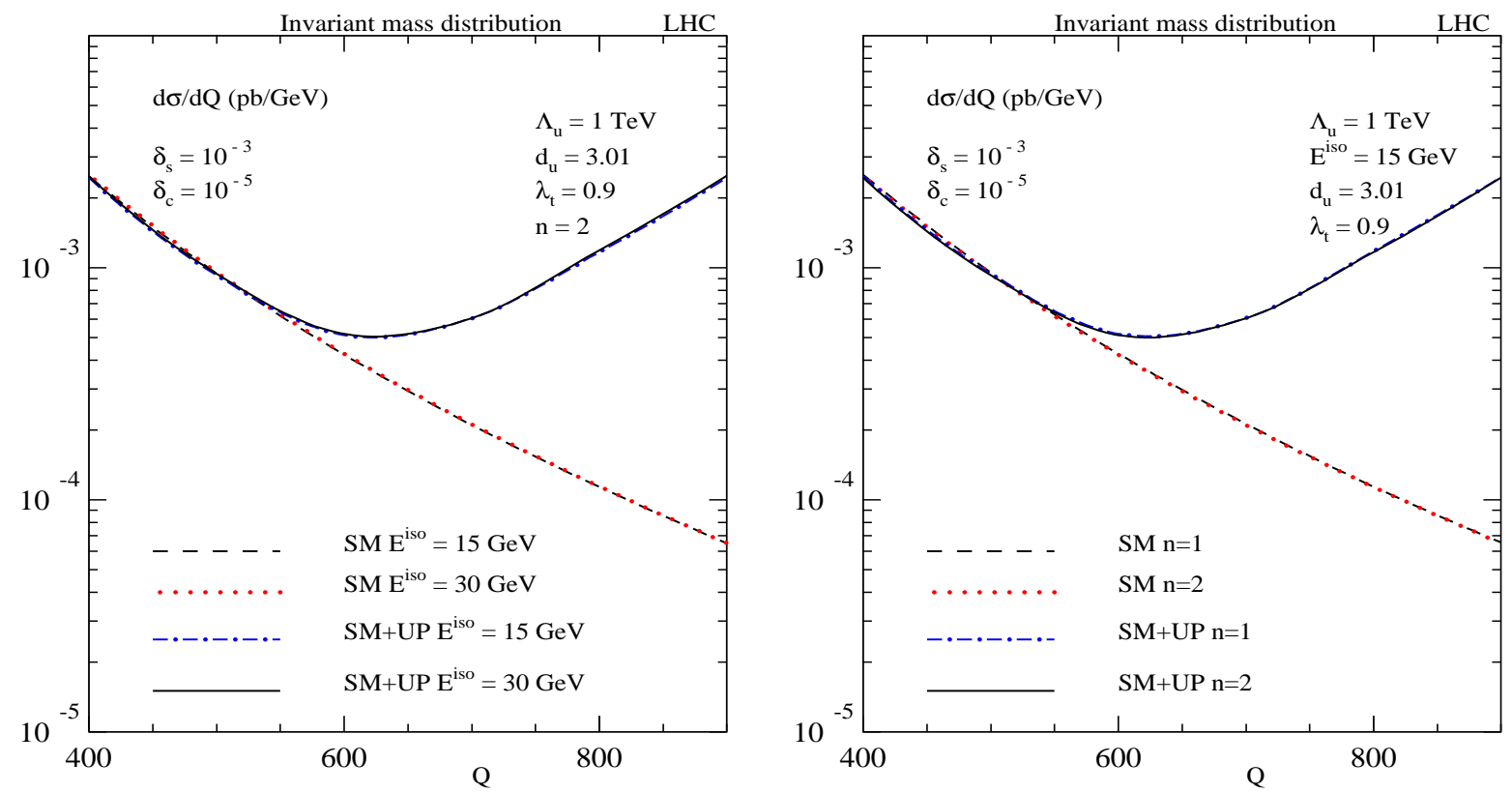

Figure 4: Dependence on isolation parameters $E_{T}^{\text {iso }}$ (left panel) and $n$ (right panel) is shown in invariant mass distribution for the range $400<Q<900 \mathrm{GeV}$.

and keeping $E_{T}^{\text {iso }}$ fixed at a value of $15 \mathrm{GeV}$. These variations are shown in Fig. 4 and show a very small dependence for $R_{0}=0.4$.

Until now our analysis was restricted to the case where $3<d_{\mathcal{U}}<4$, which was essential for a tensor unparticle as a consequence of scale invariance. There are no known examples of unitary quantum field theory that are scale invariant but not conformal invariant. For conformal invariance, unitarity demands that $d_{\mathcal{U}}>4$ for the tensor unparticles. In Fig. 5 (left panel) the unparticle sector as a result of scale invariance and not conformal $\left(d_{\mathcal{U}}=3.01\right)$ is contrasted to the case where the unparticle sector is conformal $\left(d_{\mathcal{U}}=4.001\right)$. This is to both LO and NLO in QCD and for $\Lambda_{\mathcal{U}}=1 \mathrm{TeV}$. In Fig. 5 (right panel) we have considered the invariant mass distribution for $d_{\mathcal{U}}>4$ to LO in QCD. For this plot we have considered $\Lambda_{\mathcal{U}}=2 \mathrm{TeV}$ and have probed $Q<0.9 \Lambda_{\mathcal{U}}$. Closer to $d_{\mathcal{U}}=4$ there could still be sufficient unparticle contribution for the tensor operator. The turn-around with energy behaviour of the unparticle effects is a typical feature of any physics beyond the SM. One observes a similar behaviour in models with 

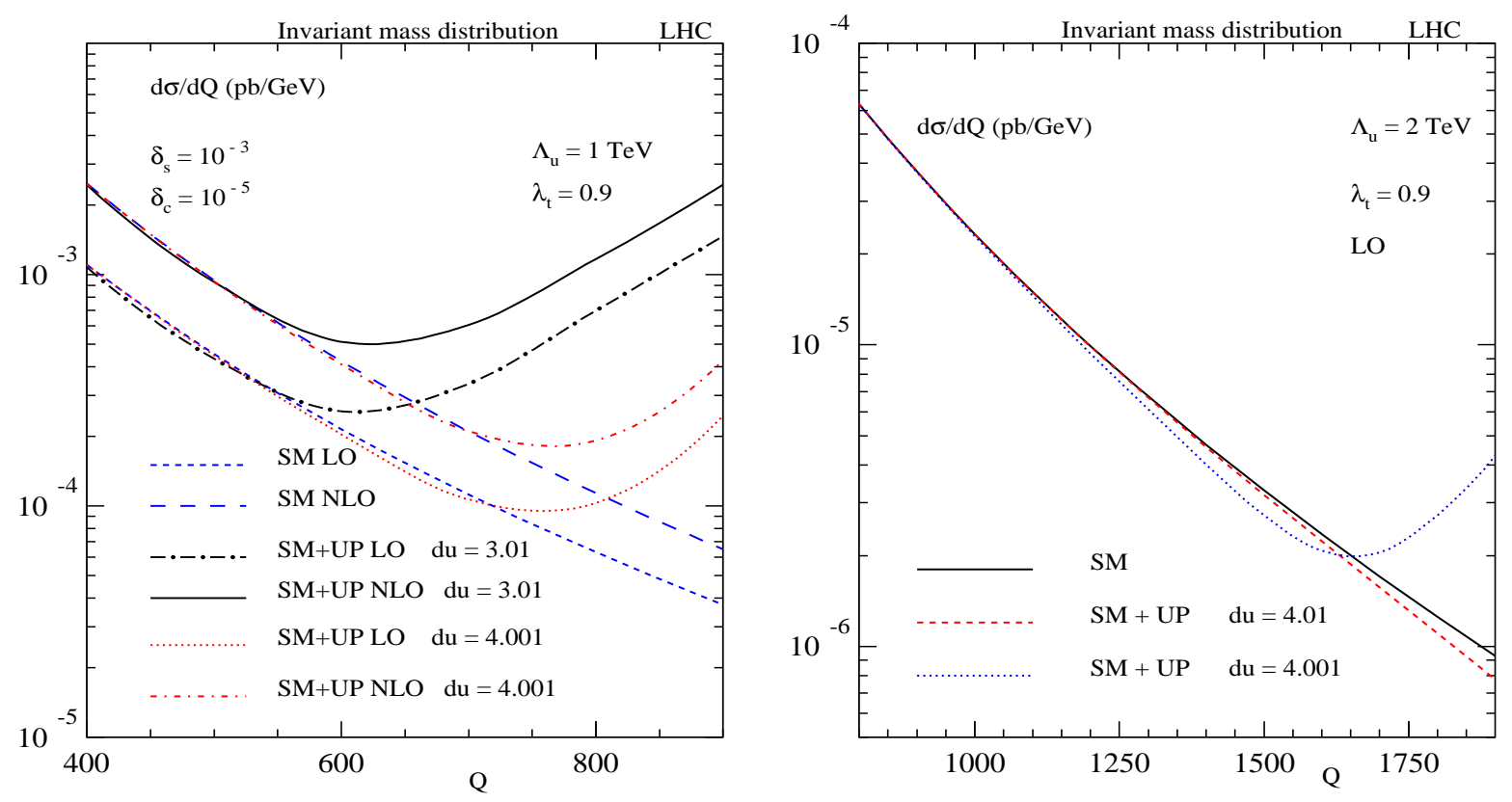

Figure 5: Invariant mass distribution of the diphoton system (left panel) for $d_{\mathcal{U}}>4$ (conformal invariance) is contrasted with the $3<d_{\mathcal{U}}<4$ (scale invariance). To LO in QCD and for $\Lambda_{\mathcal{U}}=2 \mathrm{TeV}$ (right panel), we have plotted the invariant mass distribution of the diphoton system for $d_{\mathcal{U}}>4$.

large extra-dimensions. The origin of this turn around contribution comes from the terms proportional to $\left(s / \Lambda_{\mathcal{U}}\right)^{d_{\mathcal{U}}}$ in the matrix elements involving unparticles. Finally the observable we have considered for the diphoton process does not distinguish other beyond standard model contact interactions.

\section{Conclusions}

In this article we have used one of the important channels namely the diphoton production in order to explore the scale invariant unparticle sector coupled to the SM. We have restricted ourselves to contributions coming from the tensor unparticles. Since the QCD plays an important role at the LHC, we have systematically included all the partonic subprocesses that enter at the NLO level in the strong coupling constant $\alpha_{s}$. We have employed smooth cone isolation criteria advocated by Frixione in order to reduce fragmentation contributions and thereby removing final state QED singularities. We have 
applied appropriate cuts on the photon kinematics that are used by the ATLAS Collaboration. Since it is hard to perform a fully analytic computation with isolation criteria and experimental cuts on the final state photons, we have resorted to the two cut-off phase space slicing method. We have also shown that our results are insensitive to the slicing parameters that bear no physics but are introduced in the intermediate stages of the computation. Our SM results are in agreement with the ones in the literature. We have presented two important distributions, namely, the invariant mass and the rapidity distributions of the diphoton system with the above isolation criteria and the relevant experimental cuts. For $\Lambda_{\mathcal{U}}=1 \mathrm{TeV}$, we have presented various subprocess contributions to the signal in the invariant mass distribution and found that the $g g$ contribution is dominant at high $Q$ values where the unparticle effects are visible. Both in the SM and in the unparticle case, the NLO contributions resulting from $\alpha_{s}$ enhance the cross sections, thanks to the large gluon flux at the LHC. Our NLO results to the diphoton production including the unparticle effects not only estimate the corrections from QCD to the leading contributions but also reduce the scale uncertainties coming from the factorization scale [19]. We have also considered the case where the unparticle sector is a result of scale invariance and not conformal $\left(3<d_{\mathcal{U}}<4\right)$ and also the case where it is due to conformal invariance $\left(d_{\mathcal{U}}>4\right)$ for the tensor unparticle operator.

Acknowledgments: We thank B.W. Harris for providing his code in which the phasespace slicing method is implemented. MCK would like to thank CSIR, New Delhi for financial support. The work of VR and AT has been partially supported by funds made available to the Regional Centre for Accelerator-based Particle Physics (RECAPP) by the Department of Atomic Energy, Govt. of India. AT and VR would like to thank the cluster computing facility at Harish-Chandra Research Institute where a part of the computational work for this study was carried out. We thank the referee for all the suggestions. 


\section{References}

[1] M. M. Nojiri et al., arXiv:0802.3672 [hep-ph].

[2] O. J. P. Eboli et al., Phys. Rev. D 61 (2000) 094007.

[3] M. C. Kumar et al., Phys. Rev. D 77 (2008) 055013.

[4] P. Aurenche et al., Z. Phys. C 29 (1985) 459; T. Binoth et al., Eur. Phys. J. C 16 (2000) 311.

[5] Z. Bern et al., Phys. Rev. D 66 (2002) 074018;

[6] C. Balazs et al., Phys. Rev. D 76 (2007) 013009; P. Nadolsky et al., Phys. Rev. D 76 (2007) 013008.

[7] H. Georgi, Phys. Rev. Lett. 98 (2007) 221601.

[8] H. Georgi, Phys. Lett. B 650 (2007) 275.

[9] L. Bourhis, M. Fontannaz and J. P. Guillet, Eur. Phys. J. C 2 (1998) 529.

[10] S. Frixione, Phys. Lett. B 429 (1998) 369.

[11] M. A. Stephanov, Phys. Rev. D 76 (2007) 035008.

[12] P. J. Fox et al., Phys. Rev. D 76 (2007) 075004; A. Delgado et al., JHEP 0710 (2007) 094; M. Bander et al., Phys. Rev. D 76 (2007) 115002.

[13] K. Cheung, W. Y. Keung and T. C. Yuan, Phys. Rev. Lett. 99 (2007) 051803.

[14] P. Mathews and V. Ravindran, Phys. Lett. B 657 (2007) 198.

[15] J. L. Feng et al., arXiv:0801.1534 [hep-ph] and the reference therein.

[16] B. Grinstein, K. A. Intriligator and I. Z. Rothstein, Phys. Lett. B 662 (2008) 367; G. Mach, Commun. Math. Phys. 55 (1977) 1. 
[17] T. Banks and A. Zaks, Nucl. Phys. B 196 (1982) 189.

[18] B. W. Harris and J. F. Owens, Phys. Rev. D 65 (2002) 094032.

[19] M. C. Kumar, P. Mathews, V. Ravindran and A. Tripathi, in preparation.

[20] J. Pumplin et al., JHEP 0207 (2002) 012.

[21] ATLAS Collaboration, ATLAS detector and physics performance. Technical design report. Vol. 2 (1999), CERN-LHCC-99-15. 